\title{
THE WEAK DROP PROPERTY ON CLOSED CONVEX SETS
}

\author{
PEI-KEE LIN and XINTAI YU
}

(Received 11 January 1991; revised 2 September 1991)

Communicated by P. G. Dodds

\begin{abstract}
Recall a closed convex set $C$ is said to have the weak drop property if for every weakly sequentially closed set $A$ disjoint from $C$ there exists $x \in A$ such that $\operatorname{co}(\{x\} \cup C) \cap A=\{x\}$. Giles and Kutzarova proved that every bounded closed convex set with the weak drop property is weakly compact. In this article, we show that if $C$ is an unbounded closed convex set of $X$ with the weak drop property, then $C$ has nonempty interior and $X$ is a reflexive space.
\end{abstract}

1991 Mathematics subject classification (Amer. Math. Soc.): 46B20, 46B10.

Keywords and phrases: weak drop property, reflexive space, weak property $(\alpha)$.

Let $C$ be a closed convex set. (Note: in this article, $C$ always denotes a closed convex set.) A drop $D(x, C)$ determined by a point $x \notin C$ is the convex hull of the set $\{x\} \cup C$. $C$ is said to have the drop property if for every nonempty closed set $A$ disjoint from $C$, there exists a point $a \in A$ such that $D(a, C) \cap A=\{a\}$. Daneš [1] proved that if $C$ is a bounded closed convex subset of $X$ and $A$ is a closed set at positive distance from $C$, then there exists an $a \in A$ such that $D(a, C) \cap A=\{a\}$. Modifying the above result, Kutzarova and Rolewicz [5] say that a nonempty proper closed convex set $C$ has the drop property if for every nonempty closed set $A$ disjoint from $C$, there exists a point $a \in A$ such that $D(a, C) \cap A=\{a\}$. A closed convex set $C$ is said to have the weak drop property if for every weakly sequentially closed set $A$ disjoint from $C$ there is $a \in A$ such that $D(a, C) \cap A=\{a\}$. The weak drop property was introduced by Giles, Sims, and Yorke [3] (also see [4]). They proved that the unit ball has

(C) 1994 Australian Mathematical Society 0263-6115/94 \$A2.00+0.00 
the weak drop property if and only if $X$ is reflexive.

Recall that the Kuratowski measure of a closed set $D$ is the number

$\alpha(D)=\inf \{r: D$ is covered by a finite family of open sets with diameter $<r\}$.

(Hence, if $D$ is an unbounded set, then $\alpha(D)=\infty$.)

For a convex closed set $C, F(C)$ denotes the set of all bounded linear functionals $x^{*} \in X^{*}, x^{*} \neq 0$ which is bounded above on $C$. If $x^{*} \in F(C)$, and $\delta>0$, the slice $S\left(x^{*}, C, \delta\right)$ is the set $\left\{x \in C: x^{*}(x) \geq M-\delta\right\}$, where $M=\sup \left\{x^{*}(x): x \in C\right\} . C$ is said to have property $(\alpha)$ if

$$
\lim _{\delta \rightarrow 0} \alpha\left(S\left(x^{*}, C, \delta\right)\right)=0
$$

for every $x^{*} \in F(C)$. In [5], Kutzarova and Rolewicz proved that if a convex closed set $C$ has the drop property, then $C$ has property $(\alpha)$. Clearly, if $C$ has property $(\alpha)$, then for every $x^{*} \in F(C)$ and $x_{n} \in S\left(C, x^{*}, 1 / n\right),\left\{x_{n}\right\}$ contains a convergent subsequence. Hence, we say a closed convex set has weak property $(\alpha)$ if for every $x^{*} \in F(C)$, and $x_{n} \in S\left(C, x^{*}, 1 / n\right),\left\{x_{n}\right\}$ has a weakly convergent subsequence. By James' Theorem, every bounded closed convex set with weak property $(\alpha)$ is weakly compact. On the other hand, one can easily show that every weakly compact convex set has the weak drop property and weak property $(\alpha)$. In [4], Giles and Kutzarova proved that every bounded closed convex set with the weak drop property is weakly compact. In this article, we prove that if $C$ is not weakly compact, then $C$ has the weak drop property if and only if $X$ is reflexive and $C$ has the weak property ( $\alpha$ ) (in this case, $C$ cannot be bounded). First, we show that every closed convex set with the weak drop property has weak property $(\alpha)$. The proof is essentially same as the proof of (i) implies (ii) in [4, Theorem 3].

PROPOSITION 1. Let $C$ be a closed convex set. If $C$ has the weak drop property, then $C$ has weak property $(\alpha)$. Hence, if $x^{*} \in F(C)$, then $x^{*}$ attains its supremum on $C$.

PROOF. Recall a sequence $\left\{x_{n}\right\} \subseteq X \backslash C$ is called a stream if $x_{n+1} \in D\left(x_{n}, C\right) \backslash$ $\left\{x_{n}\right\}$ for all $n>1$. Clearly, every substream is a stream. Assume that $C$ does not have weak property $(\alpha)$. We shall construct a stream which does not have any weakly convergent subsequence. Since $C$ does not have weak property $(\alpha)$, there exist $x^{*} \in F(C)$ and a sequence $\left\{x_{n}\right\}$ such that

(i) $x_{n} \in S\left(x^{*}, C, 1 / 4^{n}\right)$; 
(ii) no subsequence of $\left\{x_{n}\right\}$ converges weakly.

Let $x_{o}$ be any element in $X$ such that $x^{*}\left(x_{o}\right)=M+2$ where $M=\sup \left\{x^{*}(x)\right.$ : $x \in C$. For every $n \in \mathbb{N}$, let

$$
y_{n}=\frac{1}{2^{n}} x_{o}+\sum_{i=1}^{n} \frac{1}{2^{n-i+1}} x_{i} .
$$

Then $x^{*}\left(y_{n}\right)>M+1 / 2^{n+1}$, and $\left\{y_{n}\right\}$ is a stream. If $\left\{y_{n}\right\}$ contains a subsequence $\left\{y_{n_{k}}\right\}$ which does not have any further weakly convergent subsequence, then $\left\{y_{n_{k}}\right\}$ is weakly sequentially closed and we are done. Hence, we may assume that every subsequence of $\left\{y_{n}\right\}$ has a further weakly convergent subsequence. So there exists an increasing sequence $\left(n_{k}\right)$ such that both $\left(y_{n_{k}}\right)$ and $\left(y_{n_{k}+1}\right)$ converge weakly. But $y_{n_{k}+1}=\frac{1}{2}\left(y_{n_{k}}+x_{n_{k}+1}\right)$. So $\left(x_{n_{k}+1}\right)$ converges weakly and we get a contradiction. Therefore, $C$ must have weak property $(\alpha)$.

REMARK 1. In [6], it has been proved that if $C_{1}$ and $C_{2}$ have the drop property, then $C_{1} \cap C_{2}$ has the drop property. The same argument shows if $C_{1}$ and $C_{2}$ have the weak drop property, then $C_{1} \cap C_{2}$ has the weak drop property.

In [5], it has been proved that if $C$ is a noncompact set with the drop property, then the interior of $C$ is nonempty. The same argument shows that a similar result holds if $C$ is an unbounded closed convex set with the weak drop property. We present a proof here.

THEOREM 2. If X contains a closed convex subset which is not weakly compact and which has the weak drop property, then $X$ is reflexive.

ProOf. Let $C$ be a non-compact closed convex subset of $X$ with the weak drop property. There exists a sequence $\left\{x_{n}\right\} \subseteq C$ such that $\left\{x_{n}\right\}$ does not have any weakly convergent subsequence. For any $x \notin C, y_{n}$ is defined by

$$
y_{n}=\frac{1}{2^{n}} x+\sum_{i=1}^{n} \frac{1}{2^{n-i+1}} x_{i} .
$$

We call the sequence $\left\{y_{n}\right\}$ a dyadic stream generated $x$. We claim that every dyadic stream has a nonempty intersection with $C$.

Suppose this is not true. Then $\left\{y_{n}\right\}$ is a stream. Since $\left\{x_{n}\right\}$ does not contain any weakly convergent subsequence, by the proof of Proposition 1 there is a substream of $\left\{y_{n}\right\}$ which does not have any weakly convergent subsequence. This contradicts $C$ having the weak drop property. We have proved our claim. 
For any $z \in X$ and $k \neq 0$, the homothetic operation $T_{2, k}: X \longrightarrow X$ is given by

$$
T_{z, k}(x)=z+k(x-z) .
$$

Clearly, $T_{z, k}$ is a homeomorphism for every $z \in X$ and $k \neq 0$. Let $T_{\left\{x_{1}, x_{2}, \ldots, x_{n}\right\}}=$ $T_{x_{1}, 2} T_{x_{2}, 2} \ldots T_{x_{n}, 2}$. It is easy to see that $y_{n} \in C$ if and only if $x \in T_{\left\{x_{1}, x_{2}, \ldots, x_{n}\right\}}(C)$. So

$$
X \backslash C=\bigcup_{n=1}^{\infty} T_{\left\{x_{1}, x_{2}, \ldots, x_{n}\right\}}(C) .
$$

By the Baire category theorem, $T_{\left\{x_{1}, x_{2}, \ldots, x_{n}\right\}}(C)$ contains an open set for some $n$. So $C$ has nonempty interior.

Suppose that $\operatorname{int}(C) \neq \emptyset$. Without loss of generality, we may assume that $0 \in \operatorname{int}(C)$. So $0 \in \operatorname{int}(C \cap(-C))$. By Remark $1, C \cap(-C)$ has the weak drop property. Since it is a symmetric set, by [5, Proposition 4] it is bounded. So $X$ is a reflexive space.

REMARK 2. Kutzarova and Rolewicz [5] proved the following:

(i) If $C$ is a closed convex set the with drop property, then $D(b, C)$ is closed for every $b \notin C$.

(ii) If $C_{1}$ and $C_{2}$ have property $(\alpha)$, then both $\operatorname{co}\left(C_{1}, C_{2}\right)$ and $C_{1}+C_{2}$ have property $(\alpha)$.

(iii) Suppose that $X$ is reflexive. Let $C$ be an unbounded closed convex subset of $X$. If $C$ has property $(\alpha)$ and if $\operatorname{int}(C) \neq \emptyset$, then $C$ contains a ray $\{c+\lambda b: \lambda \geq 0\}$. Moreover, for any $x \in X$, there is $\beta>0$ such that $C$ contains the ray $\{x+\lambda b: \lambda \geq \beta\}$.

The same argument shows the above statements are still true if we replace property $(\alpha)$ by weak property $(\alpha)$.

It is natural to ask whether $X$ is reflexive if $X$ contains a closed convex subset $C$ such that

(a) $C$ is not weakly compact.

(b) $C$ has the weak drop property.

The following theorem shows that this is true if $C$ satisfies the following additional assumption

(c) $\operatorname{int}(C) \neq \emptyset$.

THEOREM 3. Suppose that $X$ contains a closed convex set $C$ which satisfies the following conditions: 
(a) C is not weakly compact.

(b) $C$ has the weak drop property.

(c) $\operatorname{int}(C) \neq \emptyset$.

Then $X$ is a reflexive space.

PROOF. We claim that that $C$ is $\mathrm{w}^{*}$-closed. Suppose the claim has been proved. Without loss of generality, we may assume that $0 \in \operatorname{int}(C)$. Then $C \cap(-C)$ is a bounded $\mathrm{w}^{*}$-closed set with nonempty interior. So $X$ is reflexive. If $x^{* *} \in \partial \bar{C}^{*}$, then by Phelps Theorem [7] for any $\epsilon>0$ there are $x_{\epsilon}^{* *} \in \partial \bar{C}^{*}$ and $x^{*} \in X^{*}$ such that $\left\|x^{* *}-x_{\epsilon}^{* *}\right\|<\epsilon$ and

$$
x_{\epsilon}^{* *}\left(x^{*}\right)=\sup x^{*}(C)=\sup x^{*}\left(\bar{C}^{*}\right) .
$$

Hence, $x^{*} \in F(C)$. But $C$ has weak property $(\alpha)$; so $D=\left\{x \in C: x^{*}(x)=\right.$ $\left.\sup x^{*}(C)\right\}$ is weakly compact, and $x_{\epsilon}^{* *}$ is an element of $D \subseteq C$. Since $C$ is a closed set, this implies $C$ contains $x^{* *}$. We have proved our claim.

THEOREM 4. Let $C$ be any unbounded closed convex subset of $X$ with a nonempty interior. Then $C$ has the weak drop property if $C$ has weak property $(\alpha)$.

Proof. By Theorem 3, $X$ is reflexive. Hence, if $C$ does not have the weak drop property, then there is a weakly closed stream $\left\{x_{n}\right\}$ which is disjoint with $C$. So $\left\{x_{n}\right\}$ does not contain any weakly convergent subsequence. Since $C$ is an unbounded set with weak property $(\alpha)$, by Remark 2 (iii), there exists $b \neq 0$ such that for every $x \in X$ there is $\beta>0$ such that $C$ contains the ray $\{x+\lambda b: \lambda \geq \beta\}$. Let $\eta=\sup \left\{\beta:\left(\beta b+\left\{x_{n}: n \in \mathbb{N}\right\}\right) \cap C=\emptyset\right\}$. Note

(i) $\eta b+C \subseteq C$;

(ii) if $\eta b+x_{n} \in C$, then $\eta b+x_{m} \in C$ for every $m>n$.

Hence, if $a_{i} \geq 0, \sum_{i=1}^{n} a_{i}=1$, and $\eta b+\sum_{i=1}^{n} a_{i} x_{i} \in \operatorname{int}(C)$, then

$$
x_{n+1}+\eta b \in \operatorname{co}\left(\left\{\eta b+\sum_{i=1}^{n} a_{i} x_{i}\right\} \cup(\eta b+C)\right) \in \operatorname{int}(C) .
$$

This is impossible. So we have that $\left(\eta b+\operatorname{co}\left(x_{n}\right)\right) \cap \operatorname{int}(C)=\emptyset$. By the Hahn-Banach Theorem, there is $x^{*} \in X^{*}$ such that

$$
\inf \left\{x^{*}\left(\eta b+x_{n}\right): n \in \mathbb{N}\right\}=M=\sup \left\{x^{*}(c): c \in C\right\} .
$$


By the definition of $\eta$, there exists a sequnce $y_{n_{k}} \in C$ such that

$$
\lim _{k \rightarrow \infty}\left\|\eta b+x_{n_{k}}-y_{n_{k}}\right\|=0, \quad \text { and } \quad \lim _{k \rightarrow \infty} x^{*}\left(y_{n_{k}}\right)=M .
$$

Since $C$ has the weak property $(\alpha),\left\{y_{n_{k}}\right\}$ contains a weakly convergent subsequence. This implies $\left\{x_{n}: n \in \mathbb{N}\right\}$ has a weakly convergent subsequence. This is a contradiction.

\section{References}

[1] J. Daneš, 'A geometric theorem useful in nonlinear functional analysis', Boll. Un. Mat. Ital. 6 (1972), 369-375.

[2] J. Diestel, Sequences and series in Banach spaces (Springer-Verlag, New York Berlin Heidelberg Tokyo, 1984).

[3] J. R. Giles, B. Sims and A. C. Yorke, 'On the drop and weak drop properties for a Banach space', Bull. Austral. Math. Soc. 41 (1990), 503-507.

[4] R. Giles and D. N. Kutzarova, 'Characterisation of drop and weak drop properties for closed bounded convex sets', Bull. Austral. Math. Soc. 43 (1991), 377-385.

[5] D. N. Kutzarova and S. Rolewicz, 'Drop property for convex sets', Journal Archiv der Mathematik 56 (1991), 501-511.

[6] P. K. Lin, 'Some remarks of the drop property', Proc. Amer. Math. Soc. 115 (1992), 441-446.

[7] R. R. Phelps, 'Weak* support points of convex sets in $E^{*}$, Israel J. Math 2 (1964), 177-182.

Memphis State University

Memphis TN 38152

USA
East China Normal University

Shanghai

China 